\title{
MODĖLES ADDITIFS ET NON ADDITIFS EN ACTUARIAT
}

\section{Philippe Vincke}

De nombreux modèles, en actuariat, se basent sur l'existence d'une fonction d'utilité additive. Le but de cet article est de montrer que cette hypothèse enlève au problème traité son caractère dynamique et que la suppression de l'additivité conduit à une solution plus réaliste.

\section{INTRODUCTION}

Il arrive fréquemment, dans les applications, et notamment en actuariat, que la décision à prendre consiste en une suite de choix qui s'échelonnent dans le temps. Cette décision est donc représentée, non par une variable $x$ mais par un vecteur $\left(x_{1}, x_{2}, \ldots, x_{N}\right)$ où chaque $x_{i}$ correspond à une période différente, la politique s'étalant sur un horizon de $N$ périodes. L'attitude généralement adoptée dans ce cas consiste à construire une fonction d'utilité additive du type

$$
U\left(x_{1}, \ldots, x_{N}\right)=\sum_{i=1}^{N} U_{i}\left(x_{i}\right) \text {. }
$$

Néanmoins, le choix d'un tel modèle exige des conditions assez restrictives sur les préférences que cette fonction d'utilité est sensée représenter (en plus des conditions impliquées par l'existence de la fonction $U$ ).

Bien rares sont les travaux qui mettent en évidence les hypothèses qu'implique un tel modèle. Le but de cet article est de montrer, à l'aide d'un problème de la théorie du risque, que l'additivité de la fonction d'utilité enlève au problème traité son véritable caractère dynamique et que la suppression de cette hypothèse peut conduire à un modèle plus réaliste, fournissant une politique plus "raisonnable".

\section{UTILITE ADDITIVE - INDEPENDANCE PREFERENTIELLE}

Soit $>$ la relation représentant les préférences d'un individu dans l'ensemble des décisions $\left\{\left(x_{1}, x_{2}, \ldots, x_{N}\right)\right\}$. Soit $U$ une fonction (supposée exister) telle que

$$
\left(x_{1}, x_{2}, \ldots, x_{N}\right)>\left(x_{1}^{\prime}, x_{2}^{\prime}, \ldots, x_{N}^{\prime}\right)
$$

ssi

$$
U\left(x_{1}, x_{2}, \ldots, x_{N}\right)>U\left(x_{1}^{\prime}, x_{2}^{\prime}, \ldots, x_{N}^{\prime}\right) .
$$

L'hypothèse d'additivité consiste à supposer l'existence de fonctions $U_{i}$ telles que

$$
U\left(x_{1}, x_{2}, \ldots x_{N}\right)=\sum_{i=1}^{N} U_{i}\left(x_{i}\right)
$$


elle implique, pour la relation $>$, des propriétés qui peuvent s'exprimer en termes d'indépendance préférentielle.

Un sous-ensemble $E$ de l'ensemble $\{1,2, \ldots, N\}$ est préférentiellement indépendant (ou indépendant au sens des préférences) dans $\{1,2, \ldots, N\}$ ssi les préférences entre des politiques qui ne diffèrent que par les composantes correspondant à $K$ sont indépendantes des autres composantes. Autrement dit, $E \subset\{1,2, \ldots, N\}$ est préférentiellement indépendant dans $\{1,2, \ldots, N\}$ ssi

$$
\left(x_{1}, x_{2}, \ldots, x_{N}\right)>\left(x_{1}^{\prime}, \ldots x_{N}^{\prime}\right)
$$

et

$$
x_{i}=x_{i}^{\prime}, \quad \forall i \in\{1,2, \ldots, N\} \backslash E
$$

entraînent

$$
\left(y_{1}, y_{2}, \ldots, y_{N}\right)>-\left(y_{1}^{\prime}, \ldots y_{N}^{\prime}\right)
$$

où

$$
\left\{\begin{array}{l}
y_{i}=x_{i}, \forall i \in E, \\
y_{i}^{\prime}=x_{i}^{\prime}, \forall i \in E, \\
y_{i}=y_{i}^{\prime}, \forall i \in\{1,2, \ldots, N\} \backslash E
\end{array}\right.
$$

De manière intuitive, cela signifie que les préférences du décideur concernant le sous-ensemble $E$ de périodes ne dépendent pas de ce qui s'est passé ou de ce qui pourra se passer au cours des autres périodes.

On peut montrer aisément que l'additivité de la fonction d'utilité nécessite l'indépendance préférentielle de tout sous-ensemble $E$ de $\{1,2, \ldots, N\}$. En effet:

$$
(1) \Rightarrow \sum_{i=1}^{N} U_{i}\left(x_{i}\right)>\sum_{i=1}^{N} U_{i}\left(x_{i}^{\prime}\right)
$$

d'où, grâce à la relation (2)

$$
\sum_{i \in E} U_{i}\left(x_{i}\right)>\sum_{i \in E} U_{i}\left(x_{i}^{\prime}\right)
$$

ou encore, par les relations (3) et (4)

$$
\sum_{i \in E} U_{i}\left(y_{i}\right)>\sum_{i \in E} U_{i}\left(y_{i}^{\prime}\right)
$$

et, en vertu de la relation (5)

$$
\sum_{i=1}^{N} U_{i}\left(y_{i}\right)>\sum_{i=1}^{N} U_{i}\left(y_{i}^{\prime}\right)
$$

par conséquent,

$$
\left(y_{1}, y_{2}, \ldots, y_{N}\right)>\left(y_{1}^{\prime} \ldots, y_{N}^{\prime}\right)
$$


Sous certaines conditions concernant les espaces $X_{i}$ auxquels appartiennent les $x_{i}$, l'indépendance préférentielle de tout sous-ensemble $E$ de $\{1,2, \ldots, N\}$ est aussi suffisante pour que la fonction d'utilité soit additive. Pour plus de précision sur ce sujet, nous renvoyons le lecteur aux travaux de Debreu (1959), Fishburn (1970), Gorman (1968), Koopmans (1960), Ting (1971), ...

L'indépendance préférentielle n'est cependant pas toujours une hypothèse très réaliste. Ainsi par exemple, en supposant qu'à chaque période le décideur ait intérêt à maximiser $x_{i}$, les fonctions $U_{i}$ du modèle précédent seront croissantes. Par conséquent, parmi les deux vecteurs suivants, c'est le premier qui aura la préférence:

(1) $(3,5,2,2,3,4,3)$

(2) $(1,2,2,2,3,3,3)$

Ce résultat est naturel si ces 2 vecteurs représentent par exemple les profits réalisés par une entreprise suite à 2 investissements qui lui coûtent le même prix. Il est peut-être beaucoup moins naturel si les 2 vecteurs représentent les dividendes versés à des actionnaires à la fin de chaque année (une politique stable inspirant souvent une plus grande confiance) ou le pouvoir d'achat de la population pour 2 plans de développement différents (pour des raisons psychologiques ou politiques).

L'hypothèse de l'existence d'une fonction d'utilité additive supprime en fait le véritable caractère dynamique d'un problème puisqu'elle implique que les préférences du décideur relativement à chaque période soient indépendantes des autres périodes.

C'est ce que nous nous proposons d'illustrer ici au moyen d'un problème de la théorie du risque. Après avoir défini le problème et rappelé un modèle additif construit par FrISQUE (1974) pour le résoudre, nous présentons un modèle qui ne nécessite pas d'hypothèse d'indépendance préférentielle. La comparaison des deux modèles montre que la suppression de l'hypothèse d'additivité conduit à une politique plus stable et, à notre avis, plus réaliste.

\section{LE PROBLÈME}

Une compagnie d'assurances verse, à la fin de chaque période, des dividendes à ses actionnaires et fixe, pour la période suivante, la part de son portefeuille qu'elle engage dans un système de réassurance. Le problème consiste à déterminer une politique de "dividendes" optimale, le but de la compagnie étant de maximiser l'utilité moyenne des dividendes. Cette utilité sera sensée représenter les préférences des actionnaires. L'horizon considéré et le montant total des primes versées à la compagnie au cours de chaque période sont des données du problème. La distribution de probabilité caractérisant les sinistres à couvrir au cours de chaque période sera fixée par le modèle. 


\section{Notations}

Soit

$S_{0} \quad$ la réserve initiale,

$N \quad$ l'horizon considéré,

$S_{j} \quad$ la réserve à la fin de la période $j(j=1,2, \ldots, N)$,

$F_{j} \quad$ la distribution de probabilité qui caractérise le montant total des sinistres pendant la période $j(j=1,2, \ldots, N)$,

$P_{j} \quad$ le montant total des primes versées au cours de la période $j(j=1,2$, $\ldots, N)$,

$s_{j} \quad$ le montant des dividendes versés aux actionnaires à la fin de la période $j(j=1,2, \ldots, N)$,

$k_{j-1}$ la partie de portefeuille gérée par la compagnie durant la période $j(j=1,2, \ldots, N)$,

$U\left(s_{1}, \ldots, s_{1+k}\right)$ l'utilité des dividendes $s_{1} \ldots s_{1+k}(k=0,1, \ldots, N-1)$, $U^{s_{1} \ldots s_{j-1}}\left(s_{j} \ldots s_{j+k}\right)$ l'utilité des dividendes $s_{j} \ldots s_{j+k}$ sachant que l'on a versé les dividendes $s_{1} \ldots s_{j-1}$ au cours des périodes précédentes $(j=2, \ldots, N ; k=0,1, \ldots, N-j)$.

Comme, $\forall k \in\{0,1, \ldots N-1\}$ :

$$
U\left(s_{1}, \ldots, s_{1+k}\right)=U\left(s_{1}\right)+\sum_{l=1}^{k} U^{s_{1} \ldots s_{l}}\left(s_{1+l}\right)
$$

les préférences des actionnaires sont entièrement caractérisées dès que l'on connaît $U\left(s_{1}\right)$ et $U^{s_{1} \ldots s_{j-1}}\left(s_{j}\right), \forall j \in\{2, \ldots, N\}$

Soit encore

$U^{s_{1} \ldots s_{j-1}}\left[S_{j}\right]$ l'utilité moyenne optimale pour la période allant de la fin de la période $j$, avant le paiement de $s_{j}$, jusqu'à la fin de l'horizon, après le paiement de $s_{N}$, sachant que la réserve à la fin de la période $j$ est $S_{j}$ et que l'on a versé les dividendes $s_{1} \ldots s_{j-1}$ au cours des périodes précédentes,

$U\left[S_{1}\right]$ l'utilité moyenne optimale pour la période allant de la fin de la première période, avant le paiement de $s_{1}$, jusqu'à la fin de l'horizon, après le paiement de $s_{N}$, sachant que la réserve à la fin de la première période est $S_{1}$,

$U\left[S_{0}\right]$ l'utilité moyenne optimale pour tout l'horizon considéré, sachant que la réserve initiale est $S_{\mathbf{0}}$.

Le principe d'optimalité de la programmation dynamique permet d'écrire: $U^{s_{1} \ldots s_{j-1}\left[S_{j}\right]}=\max \left\{U^{s_{1} \ldots s_{1-1}\left(s_{j}\right)}+\int U^{s_{1} \ldots s_{j}}\left[S_{j}-s_{j}+k_{j}\left(P_{j+1}-x\right)\right] d F_{j+1}(x)\right\}$

sous les contraintes

$$
\left\{\begin{array}{l}
0 \leqslant s_{j} \leqslant S_{j} \\
0 \leqslant k_{j} \leqslant 1
\end{array}\right.
$$




\section{Modèle de Frisque}

Dans ce modèle, la distribution des sinistres et les primes sont caractérisées, $\forall j$, par:

où $p>1 \mid 2$, et $P_{j}=1$.

$$
F_{j}(x)= \begin{cases}0 & x<0 \\ p & 0 \leqslant x<2 \\ 1 & 2 \leqslant x\end{cases}
$$

D'autre part,

et, $\forall j \in\{2, \ldots, N\}$ :

$$
U\left(s_{1}\right)=s_{1}^{1 / 2}
$$

$$
U^{s_{1} \cdots s_{j-1}}\left(s_{j}\right)=v^{j-1} s_{j}^{1 / 2}
$$

Le facteur $v$ n'a pas nécessairement un lien avec le taux d'intérêt. Il peut aussi exprimer "l'impatience" des actionnaires, c'est-à-dire "le degré qui caractérise la préférence d'un paiement récent sur un paiement ultérieur" [BoRCH (1968)]. Ainsi, par exemple, soit $v<1$ et

$$
U^{s_{1} \ldots s_{j-1}}\left(s_{j}, s_{j+1}\right)=s_{j}+v s_{j+1}
$$

Il vient

$$
U^{s_{1} \ldots s_{j-1}}(x, y)-U^{s_{1} \ldots s_{j-1}}(y, x)=x(1-v)-y(1-v)
$$

expression qui sera positive ssi $x>y$ : le décideur préfère recevoir la plus grande somme en premier lieu. C'est la définition de l'impatience donnée par Koopmans (1960).

Le modèle de Frisgue (1974) conduit à la solution suivante:

$$
\left\{\begin{array}{l}
s_{j}=\frac{S_{j}}{1+K+K^{2}+\ldots+K^{N-j}}, \quad j \in\{1,2, \ldots, N\} \\
k_{j}=\left(S_{j}-s_{j}\right) \frac{p^{2}-q^{2}}{p^{2}+q^{2}}, j \in\{1,2, \ldots, N-1\} \\
k_{0}=S_{0} \frac{p^{2}-q^{2}}{p^{2}+q^{2}} \\
U^{\delta_{1} \ldots \delta_{j-1}\left[S_{j}\right]=v^{j-1}} S_{j}^{1 / 2}\left(1+K+\ldots+K^{N-j}\right)^{1 / 2}, \quad j \in\{2, \ldots N\} \\
U\left[S_{1}\right]=S_{1}^{1 / 2}\left(1+K+\ldots+K^{N-1}\right)^{1 / 2} \\
U\left[S_{0}\right]=S_{0}^{1 / 2} \sqrt{2}\left(p^{2}+q^{2}\right)^{1 / 2} \quad\left(1+K+\ldots+K^{N-1}\right)^{1 / 2}
\end{array}\right.
$$

où

$$
\begin{aligned}
K & =2 v^{2}\left(p^{2}+q^{2}\right) \\
q & =1-p .
\end{aligned}
$$




\section{Modèle non additif}

En vue de faciliter la comparaison de ce modèle avec celui de Frisque, nous définirons $F_{j}(x)$ et $P_{j}$ de la même manière que ce dernier. D'autre part, supposons que

$$
U\left(s_{1}\right)=s_{1}^{1 / 2}
$$

et $\forall j \in\{2, \ldots, N\}$,

$$
U^{s_{1} \ldots s_{j-1}}\left(s_{j}\right)=v^{j-1}\left(j s_{j}-s_{1}-s_{2}-\ldots-s_{j-1}\right)^{1 / 2}
$$

La résolution du problème à l'aide de ce modèle a été conduite d'une manière analogue à celle de Frisque. Nous ne reproduisons pas ici les calculs, qui sont assez lourds. Le lecteur intéressé les trouvera faits en détails dans VINCKE (1977).

Ce modèle conduit à la solution suivante:

$$
\left\{\begin{array}{l}
s_{j}=S_{j} \frac{j}{\left(1+L_{j}\right) B_{j}}-\left(s_{1}+\ldots+s_{j-1}\right)\left[\frac{j L_{j-1}}{\left(1+L_{j}\right) B_{j}}-\frac{1}{j}\right], j \in\{2, \ldots, N\} \\
s_{1}=\frac{S_{1}}{\left(1+L_{1}\right) B_{1}} \\
k_{j}=\left[S_{j}-s_{j}-L_{j}\left(s_{1}+\ldots+s_{j}\right)\right] \frac{p^{2}-q^{2}}{p^{2}+q^{2}}, j \in\{1,2, \ldots, N-1\} \\
k_{0}=S_{0} \frac{p^{2}-q^{2}}{p^{2}+q^{2}} \\
U^{s_{1} \ldots s_{j-1}}\left[S_{j}\right]=v^{j-1}\left(\frac{B_{j}}{1+L_{j}}\right)^{1 / 2}\left[S_{j}-L_{j-1}\left[s_{1}+\ldots+s_{j-1}\right)\right]^{1 / 2}, j \in\{2, \ldots, N\} \\
U\left[S_{1}\right]=\left(\frac{B_{1}}{1+L_{1}}\right)^{1 / 2} S_{1}^{1 / 2} \\
U\left[S_{0}\right]=\sqrt{2}\left(p^{2}+q^{2}\right)^{1 / 2}\left(\frac{B_{1}}{1+L_{1}}\right)^{1 / 2} S_{0}^{1 / 2}
\end{array}\right.
$$

où

$$
\left\{\begin{array}{l}
L_{j}=L_{j+1}+\frac{1+L_{j+1}}{j+1}, j \in\{1,2, \ldots, N-1\} \\
L_{N}=0 \\
B_{j}=j+2 v^{2}\left(p^{2}+q^{2}\right)\left(1+L_{j}\right) \frac{B_{j+1}}{1+L_{j+1}}, j \in\{1,2, \ldots, N-1\} \\
B_{N}=N .
\end{array}\right.
$$




\section{COMPARAISON DES DEUX MODËLES}

Les tableaux qui suivent permettent de comparer les valeurs numériques auxquelles conduisent les deux modèles dans différentes situations. L'horizon choisi est de 5 périodes $(N=5)$. La notation olloo signifie qu'il n'y a pas sinistre au cours des première, quatrième et cinquième périodes et qu'il y a sinistre au cours des deuxième et troisième périodes. Les nombres qui apparaissent dans les tableaux doivent être multipliés par $S_{0}$.

TABLEAU $1: p=.7 ; q=.3 ; v=.2$

\begin{tabular}{|c|c|c|c|c|c|c|c|c|}
\hline & \multicolumn{2}{|c|}{01100} & \multicolumn{2}{|c|}{01111} & \multicolumn{2}{|c|}{11100} & \multicolumn{2}{|c|}{ ooooo } \\
\hline & Frisque & $\begin{array}{c}\text { non } \\
\text { additif }\end{array}$ & Frisque & $\begin{array}{c}\text { non } \\
\text { additif }\end{array}$ & Frisque & $\begin{array}{c}\text { non } \\
\text { additif }\end{array}$ & Frisque & $\begin{array}{c}\text { non } \\
\text { additif }\end{array}$ \\
\hline$h_{0}$ & .690 & .690 & .69 & .69 & .69 & .69 & .69 & .69 \\
\hline$S_{1}$ & 1.690 & 1.690 & 1.69 & 1.69 & $\cdot 31$ & .31 & 1.69 & 1.69 \\
\hline$s_{1}$ & 1.610 & .490 & 1.61 & .49 & $\cdot 3$ & .09 & 1.61 & .49 \\
\hline$k_{1}$ & .050 & $.15^{\circ}$ & .05 & .15 & .009 & .028 & .05 & .15 \\
\hline$S_{2}$ & .030 & 1.050 & .03 & 1.05 & .001 & .192 & .13 & 1.35 \\
\hline$s_{2}$ & .028 & .272 & .028 & .272 & .00095 & .0488 & .124 & .4 \\
\hline$k_{2}$ & .0009 & .014 & .0009 & .014 & .00003 & .0032 & .004 & .032 \\
\hline$S_{3}$ & .001 & .76 & .0011 & .76 & .00002 & .14 & .01 & .982 \\
\hline$s_{3}$ & .001 & .25 & .001 & .25 & .000019 & .046 & .0095 & .35 \\
\hline$k_{3}$ & .00003 & .009 & .00003 & .009 & .0000006 & .0018 & .0003 & .017 \\
\hline$S_{4}$ & .000133 & .519 & .000067 & .5 & .0000016 & .096 & .00075 & .65 \\
\hline$s_{4}$ & .000127 & .261 & .000064 & .255 & .0000015 & .05 & .0007 & .33 \\
\hline$k_{4}$ & .000004 & .0005 & .000002 & .00025 & .000000048 & .00027 & .00002 & .003 \\
\hline$S_{5}$ & .00001 & .25 & .000001 & .246 & $.00000014^{8}$ & .046 & .00072 & $.3^{2} 3$ \\
\hline$s_{5}$ & .00001 & .25 & .000001 & .246 & $.00000014^{8}$ & .046 & .00072 & .323 \\
\hline
\end{tabular}

TABLEAU $2: p=.7 ; q=.3 ; v=.5$

\begin{tabular}{|c|c|c|c|c|c|c|c|c|}
\hline & \multicolumn{2}{|c|}{01100} & \multicolumn{2}{|c|}{01111} & \multicolumn{2}{|c|}{11100} & \multicolumn{2}{|c|}{00000} \\
\hline & Frisque & $\begin{array}{l}\text { non } \\
\text { additif }\end{array}$ & Frisque & $\begin{array}{l}\text { non } \\
\text { additif }\end{array}$ & Frisque & $\begin{array}{c}\text { non } \\
\text { additif }\end{array}$ & Frisque & $\begin{array}{c}\text { non } \\
\text { additif }\end{array}$ \\
\hline$k_{0}$ & .69 & .69 & .69 & .69 & .69 & .69 & .69 & .69 \\
\hline$S_{1}$ & 1.69 & 1.69 & 1.69 & 1.69 & .31 & .31 & 1.69 & 1.69 \\
\hline$s_{1}$ & 1.2 & .177 & 1.2 & .177 & .22 & .032 & 1.2 & .177 \\
\hline$k_{1}$ & .33 & .79 & .33 & .79 & .06 & .145 & .33 & .79 \\
\hline$S$ & .16 & .723 & .16 & .723 & .03 & .133 & .82 & 2.3 \\
\hline$s_{2}$ & .11 & .162 & .11 & .162 & .02 & .03 & $.5^{8}$ & $.4^{8}$ \\
\hline$k_{2}$ & .031 & .16 & .031 & .16 & .006 & .027 & .16 & .8 \\
\hline$S_{3}$ & .019 & .4 & .019 & .4 & .004 & .076 & .4 & 2.62 \\
\hline$s_{3}$ & .014 & .14 & .014 & .14 & .003 & .026 & $\cdot 3$ & 1.01 \\
\hline$k_{3}$ & .0035 & .016 & .0035 & .016 & .00075 & .0025 & .075 & $.53^{6}$ \\
\hline$S_{4}$ & .0085 & .276 & .0015 & .244 & .00175 & .0525 & .175 & 2.14 \\
\hline$s_{4}$ & .0066 & .14 & .0011 & .12 & .00135 & .026 & .135 & 1.17 \\
\hline$k_{4}$ & .0013 & .01 & .0002 & .002 & .00027 & .002 & .027 & .33 \\
\hline$S_{5}$ & .0032 & .126 & .0002 & .122 & .00067 & .0285 & .067 & 1.3 \\
\hline$s_{5}$ & .0032 & .126 & .0002 & .122 & .00067 & .0285 & .067 & 1.3 \\
\hline
\end{tabular}


TABLEAU $3: p=.7 ; q=.3 ; v=.9$

\begin{tabular}{|c|c|c|c|c|c|c|c|c|}
\hline & \multicolumn{2}{|c|}{01100} & \multicolumn{2}{|c|}{01111} & \multicolumn{2}{|c|}{11100} & \multicolumn{2}{|c|}{ 00000 } \\
\hline & Frisque & $\begin{array}{l}\text { non } \\
\text { additif }\end{array}$ & Frisque & $\begin{array}{c}\text { non } \\
\text { additif }\end{array}$ & Frisque & $\begin{array}{l}\text { non } \\
\text { additif }\end{array}$ & Frisque & $\begin{array}{l}\text { non } \\
\text { additif }\end{array}$ \\
\hline$k_{0}$ & .69 & .69 & .69 & .69 & .69 & .69 & .69 & .69 \\
\hline$S_{1}$ & 1.69 & 1.69 & 1.69 & 1.69 & .31 & .31 & 1.69 & 1.69 \\
\hline$s_{1}$ & $.3^{8}$ & .0236 & $.3^{8}$ & .0236 & .07 & .0043 & $\cdot 3^{8}$ & .0236 \\
\hline$k_{1}$ & .89 & 1.115 & .89 & 1.115 & .164 & .2 & .89 & 1.115 \\
\hline$S_{2}$ & .42 & .55 & .42 & .55 & .076 & .1 & 2.96 & 2.78 \\
\hline$s_{2}$ & .115 & .042 & .115 & .042 & .02 & .0076 & .811 & .175 \\
\hline$k_{2}$ & .21 & .3 & .21 & .3 & .038 & .054 & 1.48 & 1.64 \\
\hline$S_{3}$ & .095 & .2 & .095 & .2 & .018 & .038 & 3.83 & $4 \cdot 245$ \\
\hline$s_{3}$ & .033 & .04 & .033 & .04 & .0064 & .0075 & 1.29 & .624 \\
\hline$k_{\mathrm{s}}$ & .0422 & .075 & .0422 & .075 & .008 & .014 & 1.61 & 2.2 \\
\hline$S_{4}$ & .1 & .235 & .02 & .085 & .0196 & .045 & 3.95 & 5.8 \\
\hline$S_{4}$ & .053 & .089 & .01 & .038 & .01 & .017 & 2 & 2.06 \\
\hline$k_{4}$ & .034 & .0819 & .0068 & .013 & .0066 & .0142 & 1.34 & 2.18 \\
\hline$S_{5}$ & .081 & .228 & .0032 & .034 & .016 & .0422 & 3.29 & 5.92 \\
\hline$s_{3}$ & .081 & .228 & .0032 & .034 & .016 & .0422 & 3.29 & 5.92 \\
\hline
\end{tabular}

TABLEAU $4: p=.7 ; q=.3 ; v=1.5$

\begin{tabular}{|c|c|c|c|c|c|c|c|c|}
\hline & \multicolumn{2}{|c|}{01100} & \multicolumn{2}{|c|}{01111} & \multicolumn{2}{|c|}{11100} & \multicolumn{2}{|c|}{ ooooo } \\
\hline & Frisque & $\begin{array}{c}\text { non } \\
\text { additif }\end{array}$ & Frisque & $\begin{array}{c}\text { non } \\
\text { additif }\end{array}$ & Frisque & $\begin{array}{c}\text { non } \\
\text { additif }\end{array}$ & Frisque & $\begin{array}{c}\text { non } \\
\text { additif }\end{array}$ \\
\hline$k_{0}$ & .69 & .69 & .69 & .69 & .69 & .69 & .69 & .69 \\
\hline$S_{1}$ & 1.69 & 1.69 & 1.69 & 1.69 & .31 & .31 & 1.69 & 1.69 \\
\hline$s_{1}$ & .014 & .0006 & .014 & .0006 & .0026 & .0001 & .014 & .0006 \\
\hline$k_{1}$ & 1.15 & 1.16 & 1.15 & 1.16 & .21 & .214 & 1.15 & 1.16 \\
\hline$S_{2}$ & .526 & .53 & .526 & .53 & .097 & .096 & 2.82 & 2.85 \\
\hline$s_{2}$ & .005 & .0026 & .005 & .0026 & .001 & .00045 & .03 & .0123 \\
\hline$k_{2}$ & .357 & .359 & .357 & .359 & .066 & .065 & 1.91 & 1.949 \\
\hline$S_{\mathbf{3}}$ & .164 & .168 & .164 & .168 & .03 & .03 & $4 \cdot 7$ & 4.78 \\
\hline$s_{3}$ & .02 & .006 & .02 & .006 & .0036 & .001 & .56 & .144 \\
\hline$k_{3}$ & .098 & .1 & .098 & .1 & .018 & .0196 & 2.82 & 3.146 \\
\hline$S_{4}$ & .242 & .26 & .046 & .062 & .0444 & .0486 & 6.96 & 7.782 \\
\hline$s_{4}$ & .0726 & .045 & $.013^{8}$ & .0115 & .0133 & .0085 & 2 & 1.346 \\
\hline$k_{4}$ & .116 & .137 & .022 & .031 & .02 & .025 & $3 \cdot 34$ & 4.23 \\
\hline$S_{5}$ & .28 & $.35^{2}$ & .01 & .02 & .05 & .065 & 8.3 & 10.6 \\
\hline$s_{5}$ & .28 & $.35^{2}$ & .01 & .02 & .05 & .065 & 8.3 & 10.6 \\
\hline
\end{tabular}


TABLEAU $5: p=.9 ; q=.1 ; v=.5$

\begin{tabular}{|c|c|c|c|c|c|c|c|c|}
\hline & \multicolumn{2}{|c|}{01100} & \multicolumn{2}{|c|}{01111} & \multicolumn{2}{|c|}{11100} & \multicolumn{2}{|c|}{00000} \\
\hline & Frisque & $\begin{array}{l}\text { non } \\
\text { additif }\end{array}$ & Frisque & $\begin{array}{l}\text { non } \\
\text { additif }\end{array}$ & Frisque & $\begin{array}{c}\text { non } \\
\text { additif }\end{array}$ & Frisque & $\begin{array}{l}\text { non } \\
\text { additif }\end{array}$ \\
\hline$k_{0}$ & .975 & .975 & .975 & .975 & .975 & .975 & .975 & .975 \\
\hline$S_{1}$ & 1.975 & 1.975 & 1.975 & 1.975 & .025 & .025 & 1.975 & 1.975 \\
\hline$s_{1}$ & 1.185 & .15 & 1.185 & .15 & .015 & .0019 & 1.185 & .15 \\
\hline$k_{1}$ & .77 & 1.48 & .77 & 1.48 & .00975 & .01875 & .77 & $1.4^{8}$ \\
\hline$S_{2}$ & .02 & .345 & .02 & .345 & .00025 & .00435 & 1.56 & $3 \cdot 3$ \\
\hline$s_{2}$ & .012 & .082 & .012 & .082 & .00015 & .001 & .936 & .62 \\
\hline$k_{2}$ & .0078 & .034 & .0078 & .034 & .0000975 & .00036 & .6 & 1.82 \\
\hline$S_{3}$ & .0002 & .23 & .0002 & .23 & .0000025 & .003 & 1.22 & $4 \cdot 5$ \\
\hline$s_{3}$ & .000128 & .076 & .000126 & .076 & .0000015 & .001 & .77 & 1.43 \\
\hline$k_{3}$ & .000072 & .003 & .000072 & .003 & .0000009 & o & .44 & 1.9 \\
\hline$S_{4}$ & .000146 & .157 & .000002 & .15 & .0000019 & .002 & .89 & 4.97 \\
\hline$s_{4}$ & .0001 & .0733 & .0000014 & .07 & .0000013 & .00097 & .63 & 2.54 \\
\hline$k_{4}$ & .00004 & .0025 & .0000005 & o & .0000005 & .00001 & .25 & 1.44 \\
\hline$S_{5}$ & .000086 & .08 & .0000001 & .08 & .0000011 & .001 & .51 & 3.87 \\
\hline$s_{5}$ & .000086 & .08 & .0000001 & .08 & .0000011 & .001 & .51 & 3.87 \\
\hline
\end{tabular}

Ces tableaux permettent de voir:

- que les variations de dividendes d'une période à l'autre sont beaucoup moins fortes dans le modèle non additif que dans le modèle de Frisque: la suppression de l'hypothèse d'additivité (de l'indépendance préférentielle) conduit à une politique plus stable;

- que les dividendes sont plus petits dans le modèle 2 que dans le modèle 1 en début de politique et deviennent plus grands par la suite: le modèle non additif conduit à une politique plus prudente;

- que la part du portefeuille que l'on engage dans un système de réassurance est moins importante dans le modèle 2 que le modèle 1 ;

- que le facteur $v$ joue un rôle prépondérant dans la façon de distribuer les dividendes: plus $v$ est petit, plus les dividendes sont grands en début de politique;

- que les différences entre les modèles 1 et 2 sont d'autant plus grandes que $v$ est petit: la forme de la fonction d'utilité dans le modèle non additif a un effet opposé à celui de $v$ lorsque celui-ci est petit;

- que lorsque la probabilité de sinistre est petite ( $q$ petit), la politique de dividendes est fortement influencée par la réalisation d'un sinistre (comparaison des tableaux 2 et 5 ).

\section{CONCLUSION}

Comme nous l'avons dit précédemment, l'attitude généralement adoptée, lorsqu'on cherche une politique s'étalant sur plusieurs périodes, consiste à se 
baser sur une fonction d'utilité additive, ce qui exige des hypothèses très fortes et très restrictives. Le modèle non additif présenté ici n'a pas la prétention d'être le plus adéquat pour le problème posé mais il montre à notre avis que la suppression de l'additivité conduit à une solution plus raisonnable et plus réaliste.

De manière générale, nous pensons qu'une attention plus soutenue devrait être consacrée aux hypothèses qu'entraîne le choix d'un modèle mathématique en vue d'un problème d'actuariat, non seulement pour ce qui concerne les aspects purement actuariels du problème (distributions des sinistres, primes, ...) mais aussi pour la détermination des fonctions à maximiser, qui sont sensées représenter les préférences du décideur.

\section{BIBLIOGRAPHIE}

Borch, K. (1974). The Mathematical Theory of Insurance, Lexington Books.

Borch, K. (1968). The Economics of Uncertainty. Princeton: Princeton University Press. Debreu, G. (1959). Topological Methods in Cardinal Utility Theory, dans K. J. Arrow, S. Karlin et P. SupPes (Eds., 1960), Mathematical Methods in the Social Sciences, Stanford, California: Stanford University Press.

Fishburn, P. C. (1970). Utility Theory for Decision Making, John Wiley \& Sons Inc., New York.

Frisque, A. (1974). Dynamic Model of Insurance Company's Management, Astin Bulletin, $8,57-65$.

Gorman, W. W. (1968). The Structure of Utility Functions, Review of Economic Studies, $35,367-390$.

Koopmans, T. C. (1960). Stationary Ordinal Utility and Impatience, Econometrica, 28, 287-309.

TING, M. (1971). Aggregation of attributes for multiattributed utility assessment, Technical Report 66, Operations Research Center, M.I.T.

VIncke, PH. (1976). Concept de quasi-ordve généralisé et théorèmes de représentation, Thèse de doctorat, Université Libre de Bruxelles.

Vincke, PH. (1977). Vers une généralisation des modèles de préférences utilisés en actuariat, Université Libre de Bruxelles. 